\title{
Auricular and Body Acupuncture as an Analgesic Boon in Post-surgical Chronic pain
}

\author{
Jayashree Doval and Dr Uma Hariharan* \\ Head of Department, Anesthesia, Bhagwan Mahavir Hospital, Pitampura, Delhi Government, India. \\ *Department of Anesthesia and Intensive care, Dr Ram Manohar Lohia Hospital and PGIMER, New Delhi, India.
}

Received: November 29, 2016; Accepted: December 17, 2016, Published: December 22, 2016

*Corresponding author: Uma Hariharan (MBBS, DNB, PGDHM, Fellowship Oncoanaesthesia); Assistant Professor, Dr Ram Manohar Lohia Hospital \& Post Graduate Institute of Medical Education and Research, CHS, New Delhi, India. E-mail: uma1708@gmail.com

\begin{abstract}
Acupuncture has come a long way from being a form of ancient Chinese medicine to a modern form of therapy for innumerable disorders, including chronic pain. Orthopaedic injuries can be associated with chronic pain, especially when associated with neurovascular injury or other complications. Multimodal analgesia along with surgical correction and physiotherapy is the mainstay of treatment for these types of chronic pain. We hereby present a young female patient affected by right sided radial nerve palsy following a fracture of the Olecranon and fixed with K-wiring. Her pain did not respond to medical management, but showed dramatic improvement with a series of auricular and body acupuncture sessions, along with other supplementary therapies.
\end{abstract}

Keywords: Acupuncture; Chronic Pain; Post-surgical; Olecranon fracture; Auriculotherapy; Analgesia

\section{Introduction}

Acupuncture [1] is an ancient Chinese therapy where fine needles are inserted into various points on the body. These acupoints are placed on definite meridians or energy channels on the body surface. Orthopaedic injuries [2] can be very notorious for the development of chronic pain if associated with neurovascular injury or infection or any other complication. In certain instances, chronic pain can be resistant to standard therapy. We hereby present a case of severe, intractable pain in the right arm following radial nerve injury after an elbow fracture. The pain was persistent despite surgical fracture fixation, medical treatment and physiotherapy. At this point, body acupuncture sessions were started followed by auricular acupuncture sessions, which resulted in significant reduction in pain scores.

\section{Case Report}

A twenty-seven-year-old, ASA grade 1, female patient presented to our pain clinic with severe, intractable pain of the right arm for three months. She had sustained a fracture of her right Olecranon, three months back following a fall at home. The VAS (visual analogue scale) scores at the time of presentation to the pain clinic were between 9 and 10. She also had wrist drop, with minimal movements of the right- hand fingers. Movements of the right forearm and arm were also restricted due to the pain. The pain also resulted in night time sleep disturbance and severely limited her activities of daily living. Open reduction and internal fixation with $\mathrm{K}$-wiring was done at the time of fracture under regional anaesthesia, which was uneventful. One month after the above surgery, the patient developed site infection along with pus discharge. A redo surgery was done with implant removal and pus drainage under general anaesthesia. This also did not relieve her of the severe pain. Medical management was given in the form of daily NSAID`s (non-steroidal anti-inflammatory agents), paracetamol and then tramadol (low-potency opioid), as per the WHO (world health organisation) analgesic ladder. When there was no reduction in pain scores, Gabapentin $100 \mathrm{mg}$ was added at night time for promoting an undisturbed sleep. In view of the associated nerve injury, tapering doses of steroids (oral Prednisolone) were given for a week to relieve nerve edema. Both these agents resulted in a marginal improvement in pain scores. The patient still had chronic severe pain of the right arm along with movement restriction and hence she was unable to do physiotherapy.

Acupuncture was started when her VAS pain scores were between 8 and 9. The points utilized for the first three acupuncture sittings included: $D U$ 20; Li 15; Li 4; SJ 5, H 7; and P 6 . Auriculotherapy was supplemented to acupoint therapy starting with $S F$ 4, 5 and 6, along with Shenmen point. After a period of one week from the day of starting acupuncture and concurrent auriculotherapy, the patient started showing slight improvement in finger movements and there was a reduction in pain VAS scores. At this point, the patient was started on TENS (transcutaneous electrical nerve stimulation) therapy. TENS was given at $4 \mathrm{Hertz}$ frequency and 25 milli-amperes current for duration of 30 minutes at a time, daily. After 10 sittings of combined TENS and acupuncture therapy, there was significant improvement in pain scores. This enabled the patient to perform active physiotherapy under the guidance of a trained physiotherapist. There was also improvement in performing activities of daily living and a sense 
of general well-being in patient after these acupuncture sittings. Additional acupuncture points were applied including DU20, SJ5, Li4 and Baxie points. After another week of acupuncture and TENS therapy, there was improvement in muscle power of the hand (grade 4/5) along with improvement in pain scores. Her VAS scores dropped to 1 at the end of intensive, combination therapy (See Table below). She was then advised to do regular physiotherapy and to consult the pain clinic monthly thereafter.

\section{Discussion}

Chronic pain [3] is defined as a pain persisting much beyond the usual course of an acute illness or beyond a reasonable time for the injury to heal or pain persisting for duration of three months or more. Complex regional pain syndrome (CRPS) [4] is broadly divided into two types: Type 1 (Reflex Sympathetic Dystrophy) and Type 2 (Causalgia). Complex regional pain syndrome is a form severe chronic pain with varied etiology, prolonged course and generally requiring multi-modal pain management. Causalgia occurs in patients having nerve damage at the time of injury. Our patient developed chronic pain in her right arm after right elbow fracture with radial nerve paresis. The nerve injury was probably a neurapraxia, [5] which improved in the due course of time with TENS therapy and after a course of steroids. The severe pain was resistant to medical management, hampering her dayto-day activities as well as effective physiotherapy. Hence, it was decided to start her on regular sessions of acupuncture.

Acupuncture is a form of ancient Chinese medicine, with proven effects in various diseases as well as in relieving pain (both acute and chronic). It involves insertion of fine needles into various points on the body. The various acupoints are placed on definite meridians or energy channels on the body surface. Generally, acupoints have a higher neurovascular density and are located between muscle groups. Acupuncture is based on the theory that there are two opposing and inseparable forces called yin and yang, maintaining a delicate body balance [6]. An imbalance between yin and yang leads to blockade in the flow of Qi (vital energy) along the meridians, causing different diseases. The WHO (World Health Organization) has identified more than 400 acupoints along 20 meridians on the body. The main mechanisms of analgesia [7] with acupuncture involve release of natural spinal opioids; endogenous release of steroids; increase in regional blood flow which promotes healing; gate theory of pain by Melzack; myofibrillary relaxation and balancing the mesolimbic neural pain pathway.

Auriculotherapy [8] is an extension of acupuncture therapy, with the external ear acting as the focus of stimulation. It is termed as ear or auricular acupuncture, when acupuncture needles are used for stimulation. In auriculotherapy, electrical stimulation is applied on the surface of ear reflex points. Ear acupoints can also be stimulated by manual pressure, lasers, magnets and ear pellets. Classical acupuncture identifies Yang meridians to be directly connected to the external ear. The ear is considered as a localized reflex system connected to the central nervous system. It is said to represent the whole anatomical body in an upsidedown orientation. Modern auriculotherapy is credited to Dr. Paul
Nogier of France, who discovered the sciatica point. He proposed that the ear is like an inverted fetus, with the head represented on the lower lobe, feet on the top of the external ear and the rest of the body in-between. Auriculotherapy has a definite role in treating both acute and chronic pain. Our patient was given a combination of body and ear acupuncture, in view of the severe and intractable nature of her pain.

This case highlights that acupuncture can form an integral part of multimodal pain management in complex regional pain syndromes [9] and in other forms of chronic pain. Proper acupoint selection, concurrent auriculotherapy, supplementation with TENS and physiotherapy as well as appropriate medical or surgical management form the cornerstones of success in relieving chronic pain and improving activities of daily living.

\section{Conclusions}

A combination of surgical treatment, medical management, acupuncture, auriculotherapy, TENS and physiotherapy resulted in a successful outcome in our patient. Intractable, chronic pain requires a multimodality and multi-disciplinary approach. Acupuncture is a promising pain management technique in these patients. Ear acupuncture or auriculotherapy supplements and complements the analgesic effects of body acupuncture. Both should preferably be started early during treatment for maximal benefit. The authors would like to emphasize that; these forms of therapy should not be introduced as a last resort measure in patients with severe, chronic pain. Effective physiotherapy and psychotherapy of these patients must go together, along with medical, surgical and acupuncture therapy.

\begin{tabular}{|c|c|c|}
\hline \multicolumn{3}{|c|}{$\begin{array}{l}\text { Table 1: TABLE DEPICTING STEADY IMPROVEMENT IN VAS PAIN } \\
\text { SCORES WITH SUCCESSIVE THERAPY }\end{array}$} \\
\hline $\begin{array}{c}\text { Serial } \\
\text { Number }\end{array}$ & $\begin{array}{c}\text { Treatment Given/Management } \\
\text { Stage }\end{array}$ & VAS scores \\
\hline 1. & Before start of treatment & 10 \\
\hline 2. & $\begin{array}{l}\text { After Orthopaedic surgery of the } \\
\text { Elbow }\end{array}$ & 9 \\
\hline 3. & $\begin{array}{l}\text { Following Medical management } \\
\text { (Analgesics, Steroids, Gabapentin } \\
\text { and other medications) }\end{array}$ & 8 \\
\hline 4. & Body Acupuncture & 6 \\
\hline 5. & Auriculotherapy & 4 \\
\hline 6. & $\begin{array}{c}\text { TENS (transcutaneous electrical } \\
\text { nerve stimulation) and } \\
\text { Physiotherapy sessions }\end{array}$ & 2 \\
\hline 7. & $\begin{array}{l}\text { At the end of all sittings of } \\
\text { acupuncture, auriculotherapy and } \\
\text { TENS (Total } 4 \text { weeks) }\end{array}$ & 1 \\
\hline
\end{tabular}

\section{References}

1. Vickers AJI, Cronin AM, Maschino AC, Lewith G, Mac Pherson H, Foster $\mathrm{NE}$, et al. Acupuncture for chronic pain: individual patient data metaanalysis. Arch Intern Med. 2012;172(19):1444-1453. doi: 10.1001/ archinternmed.2012.3654.

2. Rosenbloom BN, Khan S, McCartney C, Katz J. Systematic review of persistent pain and psychological outcomes following traumatic 
musculoskeletal injury. J Pain Res. 2013;6:39-51. doi: 10.2147/JPR. S38878.

3. Abu-Saad Huijer H. Chronic pain: a review.J Med Liban. 2010;58(1):2127.

4. Albazaz R, Wong YT, Homer-Vanniasinkam S. Complex regional pain syndrome: a review. Ann Vasc Surg. 2008;22(2):297-306. doi: 10.1016/j.avsg.2007.10.006.

5. Campbell WW. Evaluation and management of peripheral nerve injury. ClinNeurophysio. 2008;119(9):1951-1965. doi: 10.1016/j. clinph.2008.03.018.

6. Wu SG, He L, Wang Q, Tang YJ. An ancient Chinese wisdom for metabolic engineering: Yin-Yang. Microb Cell Fact. 2015;14:39. doi: 10.1186/s12934-015-0219-3.

7. Madsen MV, Gotzsche PC, Hrobjartsson A. Acupuncture treatment for pain: systematic review of randomised clinical trials with acupuncture, placebo acupuncture and no acupuncture groups. BMJ. 2009;338:a3115. doi: 10.1136/bmj.a3115.

8. Asher GN, Jonas DE, Coeytaux RR, Winham SJ, Reilly AC, Loh YL, et al. Auriculotherapy for Pain Management: A Systematic Review and Meta-Analysis of Randomized Controlled Trials. J Altern Complement Med. 2010;16(10):1097-1108.

9. Doval J, Hariharan U. Acupuncture as an Adjunct to Stellate Ganglion Block for CRPS. J Anesth Pain Med. 2016;1(2):1-3. 